\title{
AN EXTREMELY FAST HALO HOT SUBDWARF STAR IN A WIDE BINARY SYSTEM
}

\author{
PÉter Németh ${ }^{1}$, Eva Ziegerer ${ }^{1}$, Andreas Irrgang ${ }^{1}$, Stephan Geier $^{1,2}$, \\ Felix FÜrst ${ }^{3}$, ThOMAs Kupfer ${ }^{3,4}$, and Ulrich Heber ${ }^{1}$ \\ ${ }^{1}$ Dr. Karl Remeis-Observatory \& ECAP, Astronomical Institute, Friedrich-Alexander University Erlangen-Nuremberg, \\ Sternwartstr. 7, D-96049 Bamberg, Germany; pnemeth1981@gmail.com \\ 2 Department of Physics, University of Warwick, Coventry, CV4 7AL, UK \\ ${ }^{3}$ Division of Physics, Mathematics, and Astronomy, California Institute of Technology, Pasadena, CA 91125, USA \\ ${ }^{4}$ Department of Astrophysics/IMAPP, Radboud University Nijmegen, P.O. Box 9010, 6500 GL Nijmegen, The Netherlands \\ Received 2016 February 9; accepted 2016 March 21; published 2016 April 11
}

\begin{abstract}
New spectroscopic observations of the halo hyper-velocity star candidate SDSS J121150.27+143716.2 ( V = 17.92 mag) revealed a cool companion to the hot subdwarf primary. The components have a very similar radial velocity and their absolute luminosities are consistent with the same distance, confirming the physical nature of the binary, which is the first double-lined hyper-velocity candidate. Our spectral decomposition of the Keck/ESI spectrum provided an $\mathrm{sdB}+\mathrm{K} 3 \mathrm{~V}$ pair, analogous to many long-period subdwarf binaries observed in the Galactic disk. We found the subdwarf atmospheric parameters: $T_{\text {eff }}=30600 \pm 500 \mathrm{~K}, \log g=5.57 \pm 0.06 \mathrm{~cm} \mathrm{~s}^{-2}$, and He abundance $\log (n \mathrm{He} / n \mathrm{H})=-3.0 \pm 0.2$. Oxygen is the most abundant metal in the hot subdwarf atmosphere, and $\mathrm{Mg}$ and $\mathrm{Na}$ lines are the most prominent spectral features of the cool companion, consistent with a metallicity of $[\mathrm{Fe} / \mathrm{H}]=-1.3$. The non-detection of radial velocity variations suggest the orbital period to be a few hundred days, in agreement with similar binaries observed in the disk. Using the SDSS-III flux calibrated spectrum we measured the distance to the system $d=5.5 \pm 0.5 \mathrm{kpc}$, which is consistent with ultraviolet, optical, and infrared photometric constraints derived from binary spectral energy distributions. Our kinematic study shows that the Galactic rest-frame velocity of the system is so high that an unbound orbit cannot be ruled out. On the other hand, a bound orbit requires a massive dark matter halo. We conclude that the binary either formed in the halo or was accreted from the tidal debris of a dwarf galaxy by the Milky Way.
\end{abstract}

Key words: binaries: spectroscopic - Galaxy: halo - stars: atmospheres - stars: horizontal-branch - stars: kinematics and dynamics - subdwarfs

\section{INTRODUCTION}

The stellar population in the Galactic halo is distinct from the populations in the thin and thick disks. Without evidence for ongoing star formation, halo stars are in general metal-poor and hence much older than their disk counterparts. Those so-called population II stars also show peculiar kinematics. While disk stars are orbiting around the Galactic Center with velocities around $240 \mathrm{~km} \mathrm{~s}^{-1}$ for the solar neighborhood, the orbits of halo stars are more diverse, usually tilted against the disk and sometimes retrograde. The typical distribution for their threedimensional Galactic rest-frame velocities ( $\left.v_{\text {grf }}\right)$ ranges from about $-200 \mathrm{~km} \mathrm{~s}^{-1}$ to $+200 \mathrm{~km} \mathrm{~s}^{-1}$ (e.g., Brown et al. 2007).

Objects with even higher $v_{\text {grf }}$ and peculiar kinematics are known among halo stars. Runaway stars reach velocities of up to $\sim 300 \mathrm{~km} \mathrm{~s}^{-1}$, while the so-called hyper-velocity stars (HVS) travel with velocities that can even exceed the escape velocity of the Galaxy (Brown et al. 2005; Edelmann et al. 2005; Hirsch et al. 2005) and reach more than $\sim 1000 \mathrm{~km} \mathrm{~s}^{-1}$ (Geier et al. 2015).

Since most of the known runaway and HVS stars are young, early-type main-sequence stars, they cannot belong to the old halo population (see Brown 2015 for a review). Those objects must have been formed in the Galactic disk, accelerated, and eventually injected into the halo. However, the acceleration mechanisms still remain unclear. Runaway stars might be the remnants of binary systems where the more massive component exploded as a core-collapse supernova (SN, Blaauw 1961) or they might have been ejected by dynamical interactions in dense star clusters. HVS stars can be generated when a close binary is disrupted by the supermassive black hole $(\mathrm{SMBH})$ in the center of our Galaxy and one component is ejected (Hills 1988).

A peculiar sub-population of these extreme halo stars consists of O-type and B-type hot subdwarf stars (sdO/Bs; see Heber 2009 for a review). One of those stars (US 708, HVS 2) turned out to be the fastest unbound star in our Galaxy (Hirsch et al. 2005; Geier et al. 2015). Hot subdwarfs form if the progenitor loses its envelope almost entirely after passing the red giant branch and the remaining hydrogen-rich envelope has not retained enough mass to sustain a hydrogen-burning shell. Mass-transfer in a binary system is considered to be the most likely mechanism to remove the envelope and form a hot subdwarf (Han et al. 2002, 2003). It is challenging to explain the acceleration of such stars to high velocities, because the most plausible scenarios are only able to explain the acceleration of single stars.

Geier et al. (2015) explained the extreme velocity of US 708 by proposing that this star is the ejected companion of a very tight binary system where the massive white dwarf primary exploded as a thermonuclear type Ia SN. In this scenario masstransfer from the hot subdwarf to the white dwarf triggered the $\mathrm{SN}$ explosion. Since the properties of such ejected companions allow us to put constraints on the unknown parameters of the progenitor systems of SNe Ia, it is crucial to find more objects like US 708.

Several other hot subdwarfs with high $v_{\text {grf }}$ have been discovered by Tillich et al. (2011). The fastest object in this sample is the sdB star SDSS J121150.27+143716.2 (also PB 3877; here J1211 for short) with $v_{\text {grf }}=713 \pm 140 \mathrm{~km} \mathrm{~s}^{-1}$, 

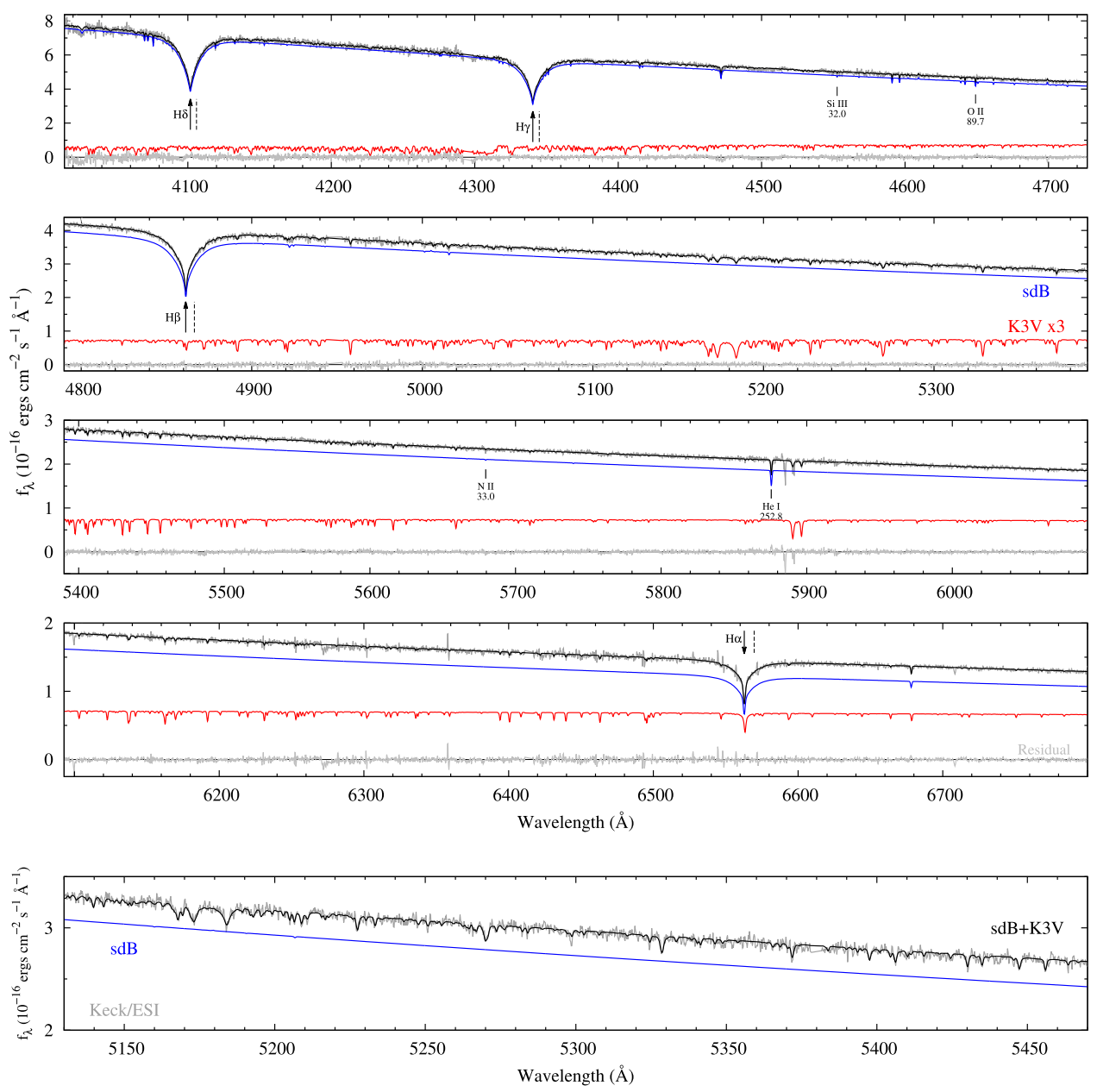

Figure 1. Top panels: radial-velocity-corrected and coadded Keck/ESI spectrum of J1211 (gray). The subdwarf (blue) dominates the spectrum in the entire range, while the K3V companion (red) contributes $17 \pm 3 \%$ of the flux at $6750 \AA$. The thin black line overplotted in the observation is the best-fit XTGRID binary model, the sum of the two components. To make the companion distinguishable from the residual its flux is multiplied by three. The flux level was adjusted to the BOSS/DR12 spectrum. The dashed lines indicate the observed wavelengths of the Balmer lines. The same shift can be observed between the Na D lines originating from the companion and from the interstellar material. The strongest $\mathrm{He}, \mathrm{N}, \mathrm{O}$, and $\mathrm{Si}$ lines are marked and labeled with their theoretical equivalent widths in m $\AA$. The atmospheric parameters of the binary members are given in Table 1. Bottom panel: zoom-in to the strongest lines of the companion in the 5150-5450 A region.

which is predominantly a tangential velocity. Tillich et al. (2011) found J1211 to most likely be unbound to the Galaxy. They traced the trajectory of this star back to the outskirts of the Galactic disk and showed that this star could not have been ejected by the SMBH in the Galactic Center. Those properties made J1211 an excellent candidate for the SN Ia ejection scenario and a prime target for our follow-up campaign with $8 \mathrm{~m}$-class telescopes.

\section{OBSERVATIONS AND SPECTROSCOPIC ANALYSIS}

To improve the atmospheric parameters of J1211 and measure the rotational broadening of the lines, we obtained medium-resolution spectra with the ESI spectrograph $(R=$ $8000,4000-6800 \AA$ ) at the Keck telescope and the XSHOOTER spectrograph $(R=10000,3000-10000 \AA)$ at the ESOVLT between 2014 June 1 and 11. To our surprise, the better resolutions and $\mathrm{S} / \mathrm{Ns}$ of these spectra, compared to the SDSS and ESO-VLT FORS1 spectra used by Tillich et al. (2011), immediately revealed the unseen weak lines of a cool companion in the spectrum.

We performed a model atmosphere analysis and fitted the composite spectra with the steepest-descent spectral analysis program XTGRID (Németh et al. 2012). This fit procedure allows the separation of the members in composite spectra binaries and employs Tlustr/SYNSPEC (Hubeny \& Lanz 1995; Lanz \& Hubeny 2007) model atmospheres for the hot subdwarf, and AtLAS9 models for the cool companion. The plane-parallel, non-LTE TLusty model atmospheres are calculated in a hydrostatic and radiative equilibrium that is appropriate for hot subdwarfs. Our models include $\mathrm{H}, \mathrm{He}, \mathrm{C}, \mathrm{N}$, $\mathrm{O}$, and $\mathrm{Si}$ opacities that are consistently in the model atmosphere calculations and in the spectral synthesis. XTGRID starts with an initial model and iteratively updates the parameters of the binary along the steepest-gradient of the global chi-squared $\left(\chi^{2}\right)$ surface. TLuSTY models are calculated in every iteration and the spectrum of the cool companion is regularly extracted from the BLUERED high-resolution synthetic stellar spectral library (Bertone et al. 2008). The BLUERED grid allows us to fit the temperature, gravity, and scaled-solar metallicity of the cool companion using interpolated models. The fit was based only on spectral lines because a reliable flux calibration was not available for the higher quality Keck and VLT data. The best fit is shown in Figure 1 and the final parameters together with error bars are listed in Table 1. For a better representation of the observed data the continuum of the 
Table 1

Parameters of the Spectroscopic Model Fit Shown in Figure 1, Photometric Model Shown in Figure 2, and Kinematic Model Shown in Figure 3

\begin{tabular}{|c|c|c|c|c|}
\hline Spectroscopic Parameters & Value & $+1 \sigma$ & $-1 \sigma$ & $\times$ Solar \\
\hline \multicolumn{5}{|l|}{$s d B$} \\
\hline$T_{\text {eff }}(\mathrm{K})$ & 30600 & 400 & 500 & $\cdots$ \\
\hline $\log g\left(\mathrm{~cm} \mathrm{~s}^{-2}\right)$ & 5.57 & 0.08 & 0.06 & $\cdots$ \\
\hline $\log n(\mathrm{He}) / n(\mathrm{H})(\mathrm{dex})$ & -3.02 & 0.20 & 0.10 & 0.009 \\
\hline $\log n(\mathrm{C}) / n(\mathrm{H})(\mathrm{dex})$ & $-4.78>$ & $\cdots$ & $\ldots$ & $0.037>$ \\
\hline $\log n(\mathrm{~N}) / n(\mathrm{H})(\mathrm{dex})$ & -4.60 & 0.16 & 0.17 & 0.370 \\
\hline $\log n(\mathrm{O}) / n(\mathrm{H})(\mathrm{dex})$ & -4.47 & 0.14 & 0.25 & 0.069 \\
\hline $\log n(\mathrm{Si}) / n(\mathrm{H})(\mathrm{dex})$ & -5.80 & 0.19 & 1.15 & 0.049 \\
\hline \multicolumn{5}{|l|}{$K 3 V:$} \\
\hline$T_{\text {eff }}(\mathrm{K})$ & 4850 & 300 & 300 & $\cdots$ \\
\hline $\log g(\operatorname{dex})$ & 4.6 & 0.4 & 0.4 & $\cdots$ \\
\hline$[M / H](\operatorname{dex})$ & -1.3 & 0.3 & 0.3 & $\cdots$ \\
\hline $\mathrm{F}_{2} / \mathrm{F}_{1}($ at $6750 \AA)$ & 0.189 & 0.039 & 0.027 & $\cdots$ \\
\hline Distance (kpc) & 5.4 & 0.5 & 0.5 & $\cdots$ \\
\hline Photometric Parameters & Value & $+1 \sigma$ & $-1 \sigma$ & \\
\hline \multicolumn{5}{|l|}{$K 3 V:$} \\
\hline$T_{\text {eff }}(\mathrm{K})$ & 4610 & 180 & 200 & \\
\hline $\log g(\operatorname{dex})$ & 4.51 & 0.14 & 0.04 & \\
\hline$M\left(M_{\odot}\right)$ & 0.7 & 0.2 & 0.0 & \\
\hline$A_{V}(\mathrm{mag})$ & 0.122 & 0.015 & 0.016 & \\
\hline Distance (kpc) & 5.68 & 0.10 & 0.09 & \\
\hline
\end{tabular}

\begin{tabular}{|c|c|}
\hline Kinematic Parameters (Model-III) & Values $\pm 1 \sigma$ \\
\hline \multicolumn{2}{|l|}{ At the current location: } \\
\hline $\begin{array}{l}\text { Heliocentric radial and tangential } \\
\text { velocity }\left(\mathrm{km} \mathrm{s}^{-1}\right) \text { : }\end{array}$ & $v_{r}=234.5 \pm 2.1 ; v_{t}=520 \pm 86$ \\
\hline $\begin{array}{l}\text { Proper motion (mas } \mathrm{yr}^{-1} \text {; Tillich } \\
\text { et al. 2011): }\end{array}$ & $\begin{array}{c}\mu_{\alpha}(\cos \delta)=-12.1 \pm \\
1.8 ; \mu_{\delta}=-27.2 \pm 1.4\end{array}$ \\
\hline Cartesian coordinates $(\mathrm{kpc})$ & $\begin{array}{c}x=-8.54 \pm 0.02 \\
y=-1.48 \pm 0.14 ; z=5.35 \pm 0.49\end{array}$ \\
\hline Cartesian velocities $\left(\mathrm{km} \mathrm{s}^{-1}\right)$ & $\begin{aligned} v_{x}=81.3 \pm 45.8 \\
v_{y}=-562.4 \pm 77.8 \\
v_{z}=26.6 \pm 21.7\end{aligned}$ \\
\hline Galactic radial velocity $\left(\mathrm{km} \mathrm{s}^{-1}\right)$ & $U=16.9 \pm 48.3$ \\
\hline Galactic rotational velocity $\left(\mathrm{km} \mathrm{s}^{-1}\right)$ & $V=-567.9 \pm 77.1$ \\
\hline Galactic rest-frame velocity $\left(\mathrm{km} \mathrm{s}^{-1}\right)$ & $v_{\text {grf }}=571.3 \pm 76.4$ \\
\hline At the last disk passage: & \\
\hline Time of disk passage (Myr) & $T=-69.5 \pm 21.1$ \\
\hline Cartesian coordinates $(\mathrm{kpc})$ & $\begin{array}{r}x=-8.3 \pm 3.3 \\
y=34.8 \pm 15.1 ; z=0.0\end{array}$ \\
\hline Cartesian velocities $\left(\mathrm{km} \mathrm{s}^{-1}\right)$ & $\begin{array}{l}\quad v_{x}=-45.2 \pm 40.1 \\
v_{y}=-420.3 \pm 48.7 \\
v_{z}=96.2 \pm 18.4\end{array}$ \\
\hline Galactic radial velocity $\left(\mathrm{km} \mathrm{s}^{-1}\right)$ & $U=-393.2 \pm 66.1$ \\
\hline Galactic rotational velocity $\left(\mathrm{km} \mathrm{s}^{-1}\right)$ & $V=-149.8 \pm 35.7$ \\
\hline Galactic rest-frame velocity $\left(\mathrm{km} \mathrm{s}^{-1}\right)$ & $v_{\mathrm{grf}}=436.5 \pm 41.6$ \\
\hline
\end{tabular}

Note. The quoted error bars are one-dimensional statistical errors. Abundances are also listed with respect to the solar mixture (Asplund et al. 2009). Note that the cylindrical galactic velocity component $W$ is equal to the Cartesian velocity component $v_{z}$.

Keck observation was adjusted to the composite model in Figure 1. No attempt has been made to include parameter correlations in the error calculations.

Our fit confirmed the sdB classification of the primary and provided a K3V type main-sequence companion. The revised sdB temperature is systematically lower by $\sim 2000 \mathrm{~K}$, while the surface gravity and He abundance are within error bars of the previous analysis by Tillich et al. (2011). The discrepancy originates from the different data set used in the analysis. Tillich et al. (2011) used the SDSS-I DR7 spectrum that shows significant differences compared to the SDSS-III, Keck, and VLT data analyzed here. We note that the $\chi^{2}$ map of a composite spectrum model resembles a valley, or canyon, with a flat bottom due to strong correlations between the atmospheric and binary parameters. Therefore, a $T_{\text {eff }}=28000 \mathrm{~K}$, $\log g=5.46 \mathrm{~cm} \mathrm{~s}^{-2}$ or a $T_{\text {eff }}=33000 \mathrm{~K}, \log g=5.67 \mathrm{~cm} \mathrm{~s}^{-2}$ solution gives only a $10 \%$ higher $\chi^{2}$ minimum. Similar degeneracies have been reported in other composite spectra binaries (Vos et al. 2013).

It is not possible to determine the orbital parameters of the binary system with the available data. We measured the radial velocities (RVs) of two SDSS spectra taken in 2005 and 2012, an ESO-VLT FORS1 spectrum taken in 2008, seven ESI spectra, and eight XSHOOTER spectra taken in 2014. No significant RV shift was detected over the timebase of nine years. Furthermore, the RV from the spectral lines of the sdB primary closely matches the one of the K-star; they both are shifted by $\sim 240 \mathrm{~km} \mathrm{~s}^{-1}$ with respect to the barycentric reference frame. A chance alignment can therefore be excluded and the two components visible in the spectra indeed form a binary system.

About $20 \%-30 \%$ of sdB stars are in composite spectrum binaries with F-G-K type companions. The orbital periods for about a dozen of these systems could be determined only recently (e.g., Vos et al. 2013). They turned out to lie between $\sim 700$ and $\sim 1300$ days, which is consistent with the Roche-lobe overflow evolution channel (Han et al. 2002). Stable mass transfer to the main-sequence companion most likely removed the envelope of the red giant and led to the formation of the $\mathrm{sdB}$ (Chen et al. 2013). Typical RV semi-amplitudes of those binaries are smaller than $20 \mathrm{~km} \mathrm{~s}^{-1}$. Although the low resolution of our data did not allow us to find RV variations, the RVs of the sdB and the K-star differ by $25 \pm 13 \mathrm{~km} \mathrm{~s}^{-1}$ in the coadded Keck spectrum, which is consistent with such wide binaries. Therefore, assuming an inclination of $i>30^{\circ}$, we expect that $\mathrm{J} 1211$ is a typical sdB+MS binary with an orbital period of several hundred days and a separation of several au. An observational confirmation will require high dispersion spectroscopy.

\section{SPECTROSCOPIC DISTANCE}

The neglect of the companion flux and the different flux calibration of the SDSS-I DR7 spectrum provided a large distance in the previous analysis. Therefore, we calculated the distance to J1211 by scaling the synthetic composite spectrum to the flux calibrated SDSS-III/BOSS DR12 observation. The spectral decomposition allowed us to measure the flux ratio $\left(F_{r}\right)$ from the composite spectrum:

$$
F_{r}=\frac{F_{2}}{F_{1}}=\frac{f_{2} R_{2}^{2}}{f_{1} R_{1}^{2}}
$$

where all flux values are monochromatic and $f_{1}$ is the flux of the subdwarf with radius $R_{1}$, and $f_{2}$ and $R_{2}$ are the surface flux and radius of the companion. We approximated the subdwarf mass with the canonical mass of $0.48 M_{\odot}$ and used the surface gravity from spectroscopy to find the radius $R_{1}=0.188 \pm 0.013 R_{\odot}$, which is a typical value for similar 


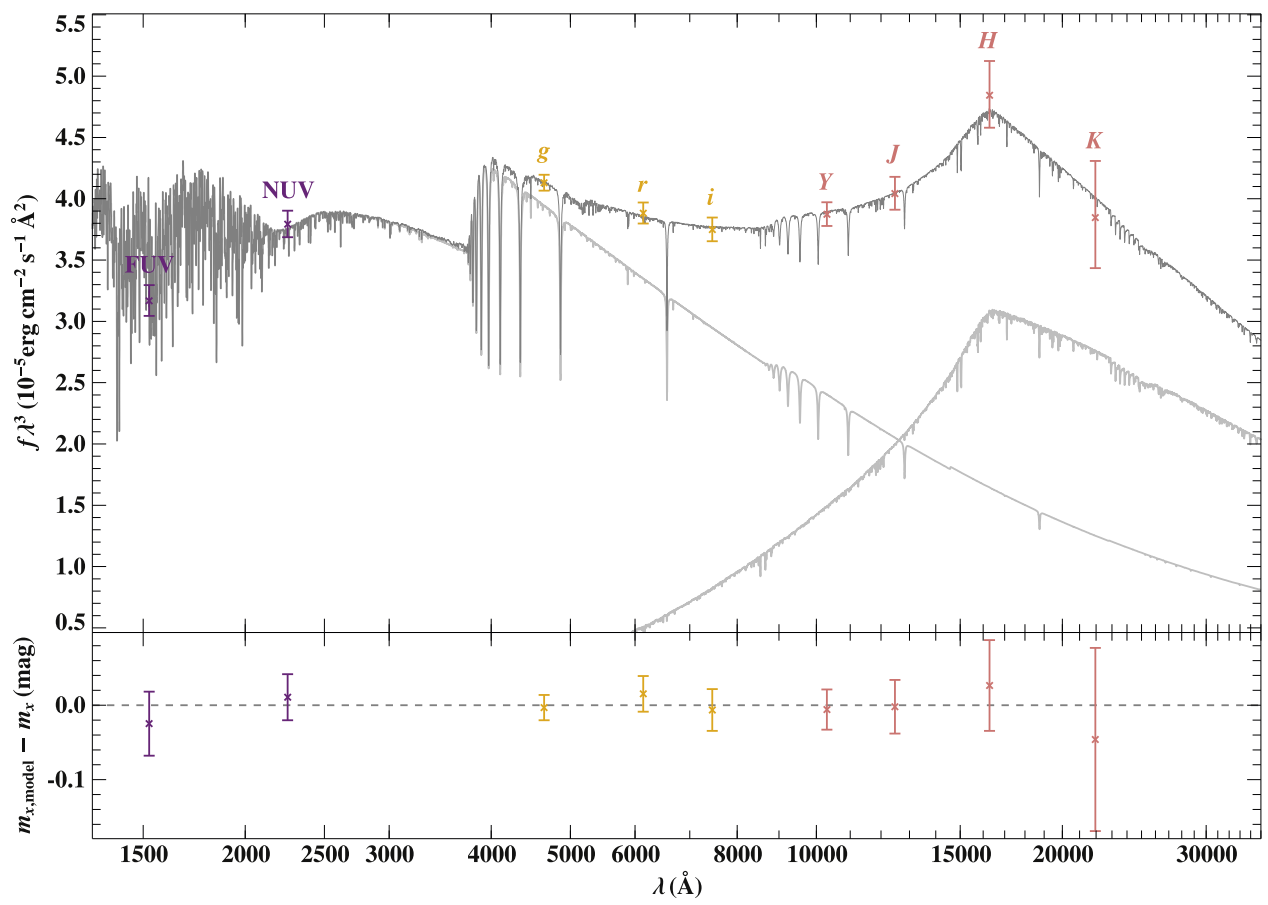

Figure 2. Comparison of synthetic and observed photometry. Top panel: the spectral energy distribution. The colored data points are fluxes, which are converted from observed magnitudes, and the solid gray line is the composite $(\mathrm{sdB}+\mathrm{K})$ model. The individual contributions are plotted in light gray. Bottom panel: the residuals show the differences between synthetic and observed magnitudes. The photometric systems have the following color code: GALEX (violet), SDSS (gold), UKIDSS (pink).

hot subdwarfs. Next, we used the surface flux of the subdwarf model to find the absolute contribution of the companion $\left(F_{2}\right)$. Then, from the ratio of the observed flux $(F)$ and the composite model, the flux scale factor $\left(F_{1}\left(1+F_{r}\right) F^{-1}\right)$ and the distance can be calculated:

$$
d=R_{1} \sqrt{\frac{F_{1}\left(1+F_{r}\right)}{F}} .
$$

The different radii of the subdwarf and the cool companion are implicitly included in the composite model and set up by the fit procedure through the flux ratio. The synthetic composite spectrum based on the Keck observation fits the slope of the BOSS spectrum well, also suggesting a relatively low reddening toward J1211 in agreement with the low interstellar extinction in its direction (see Section 4). We measured the scale factor around $5800 \AA$ where the effects of reddening, atmospheric extinction and spectral lines are the lowest in the observation. We found a scale factor of $1.66 \pm 0.05 \times 10^{24}$, which gives a spectroscopic distance of $d=5.4 \pm 0.5 \mathrm{kpc}$.

\section{PHOTOMETRIC CONSISTENCY CHECK}

To check whether the spectroscopic results are consistent with photometry, we performed a fit of the observed spectral energy distribution (SED). The latter is given by the following magnitudes compiled from the literature: GALEX DR5 $\mathrm{FUV}=16.663 \mathrm{mag}$ and $\mathrm{NUV}=17.039 \mathrm{mag}$ (Bianchi et al. 2011), $\operatorname{SDSS}^{5}$ DR12 $g=17.740 \mathrm{mag}, r=18.076$ mag, and $i=18.298 \mathrm{mag}$, UKIDSS DR9 $Y=18.039 \mathrm{mag}$, $J=17.898 \mathrm{mag}, H=17.580 \mathrm{mag}$, and $K=17.612 \mathrm{mag}$ (Lawrence et al. 2007). The fitting parameters encompass a distance scaling factor, a measure for the interstellar extinction

\footnotetext{
5 The $u$ - and $z$-magnitudes of SDSS are obvious outliers and are thus not used.
}

(parameterized here according to the description of Fitzpatrick 1999), and the surface ratio of the two stars, which is needed to weight the fluxes in the composite SED. While atmospheric parameters for the sdB TLusTY model are kept fixed and are taken from spectroscopy, the temperature and surface gravity of the cool companion are fitted with models extracted from the Phoenix grid of Husser et al. (2013) as the BlueRed grid does not cover the SED. This way we also test whether the results for the $\mathrm{K}$ dwarf are model-dependent. To enhance the sensitivity of the photometric analysis to $\log g_{2}$, we express the surface ratio, which is well-constrained because the SED covers data points dominated by the $\mathrm{sdB}$ (ultraviolet), as well as by the K-star (infrared), as a function of gravity and mass, i.e., $\left(R_{2} / R_{1}\right)^{2}=M_{2} g_{1} /\left(M_{1} g_{2}\right)$, with $M_{1}$ set to the canonical mass of $0.48 M_{\odot}$ and $\log g_{1}$ to its spectroscopic value. Given the K3V type of the companion, its mass is limited to the small range $0.7-0.9 M_{\odot}$, effectively making the surface ratio a probe for $g_{2}$. The results of the fit are listed in Table 1 and visualized in Figure 2 and show a perfect agreement with spectroscopy. The given uncertainties are statistical $1 \sigma$-confidence intervals based on the $\chi^{2}$ statistics of the residuals shown in Figure 2. Note that the offset with respect to the spectroscopic distance is mainly caused by discrepancies in the absolute flux calibration between the SDSS spectrum and photometry, which are of the order of $0.1 \mathrm{mag}$.

\section{KINEMATICS}

To obtain the dynamical properties of J1211, three different Milky Way mass models (see models I, II, and III by Irrgang et al. 2013) were used to trace back the orbit to the Galactic disk. Model-I is a revised model of Allen \& Santillan (1991) with a halo mass of $M_{\mathrm{R}<200 \mathrm{kpc}}=1.9 \times 10^{12} M_{\odot}$ within a radius of $200 \mathrm{kpc}$. For Model-II, the halo mass distribution is replaced by a truncated, flat rotation curve model according to 

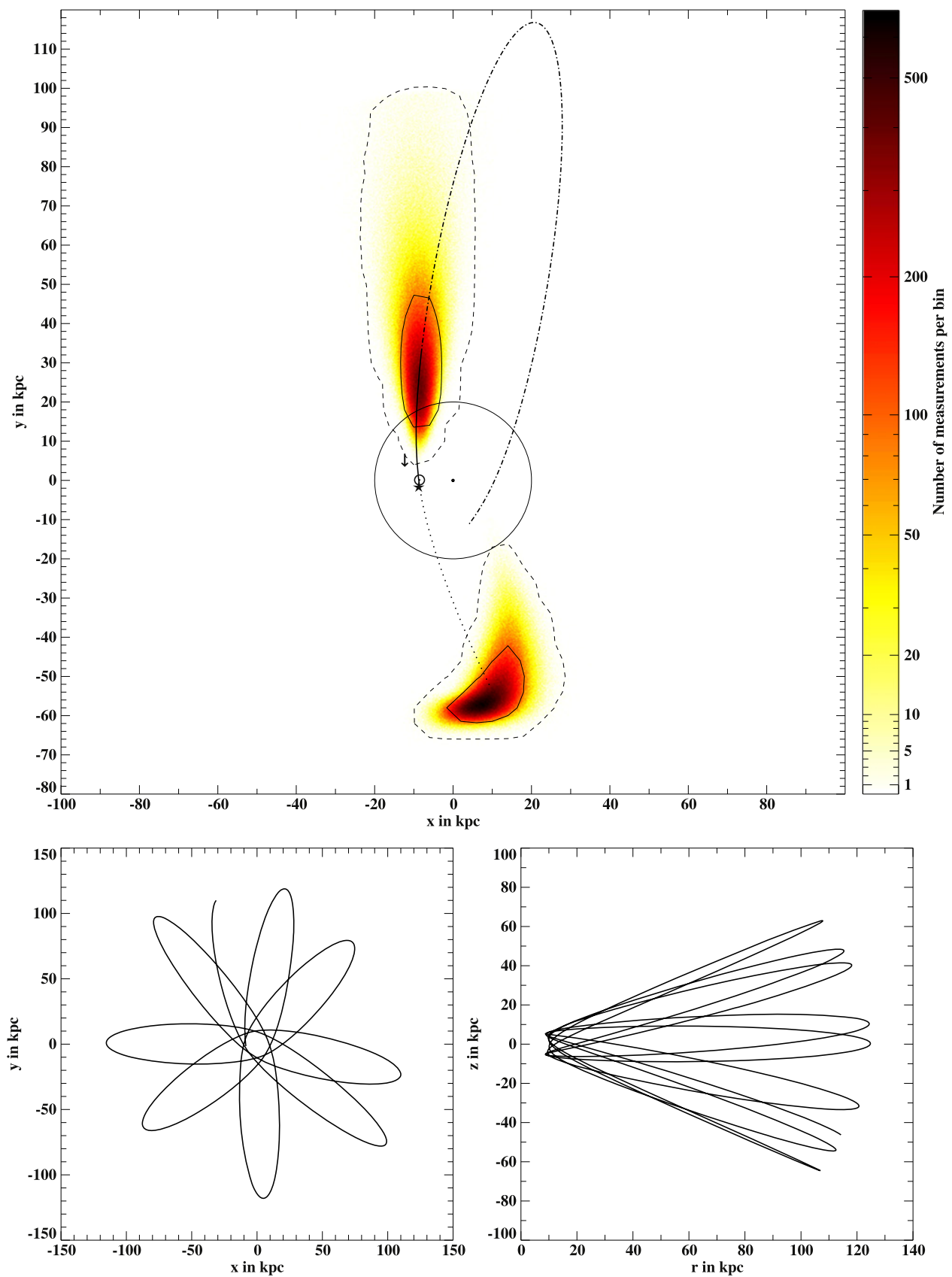

Figure 3. Top panel: disk passages (binned and color coded) of J1211 from our Monte Carlo simulation using Model-III of Irrgang et al. (2013) with $1 \sigma$ (solid) and $3 \sigma$ (dashed) contours. The solar symbol marks the position of the Sun, the black dot represents the position of the Galactic Center and the star marks the current position of J1211. The projection of one orbit onto the $x$-y-plane is shown with the dashed-dotted line for $z<0$, with a full line from $z=0$ to the current position, and with a dotted line from the current position of J1211 to the next passage of the $x$-y-plane. Bottom panels: estimated orbits of J1211 in the $x$-y-plane (left) and $r$-z-plane (right) in the most massive Galactic potential over the past $10 \mathrm{Gyr}$.

Wilkinson \& Evans (1999) and Sakamoto et al. (2003) with $M_{\mathrm{R}<200 \mathrm{kpc}}=1.2 \times 10^{12} M_{\odot}$. The dark matter halo in Model-III has $M_{\mathrm{R}<200 \mathrm{kpc}}=3.0 \times 10^{12} M_{\odot}$ and is based on the density profile by Navarro et al. (1997), derived from cosmological simulations. All mass models are the sum of a central spherical bulge component, an axisymmetric disk component and a massive spherical dark matter halo. The model parameters were recalibrated using new and improved observational constraints by Irrgang et al. (2013). The intersection area of the trajectories of J1211 with the Galactic plane and the median $v_{\text {grf }}$ at the present location were determined by varying the position and velocity components within their respective errors by applying a Monte Carlo procedure with a depth of $10^{6}$ for each mass model. The bound probability is defined as the number of orbits not exceeding the local escape velocity, i.e., the number of orbits with a negative sum of their potential and kinetic energy, with respect to the number of all calculated orbits.

Using the combined spectroscopic and photometric distance of $d=5.5 \pm 0.5 \mathrm{kpc}$ we found $\mathrm{J} 1211$ at a Galactocentric distance of $10.2 \pm 0.3 \mathrm{kpc}$ with $v_{\text {grf }}=571.3 \pm 76.4 \mathrm{~km} \mathrm{~s}^{-1}$. To reconstruct the orbit we took proper motions from Tillich et al. (2011). For the most massive mass model (Model-III) 99.7\% of the orbits are bound to the Galaxy; for the least massive mass model (Model-II) only $40.3 \%$ of the orbits are 


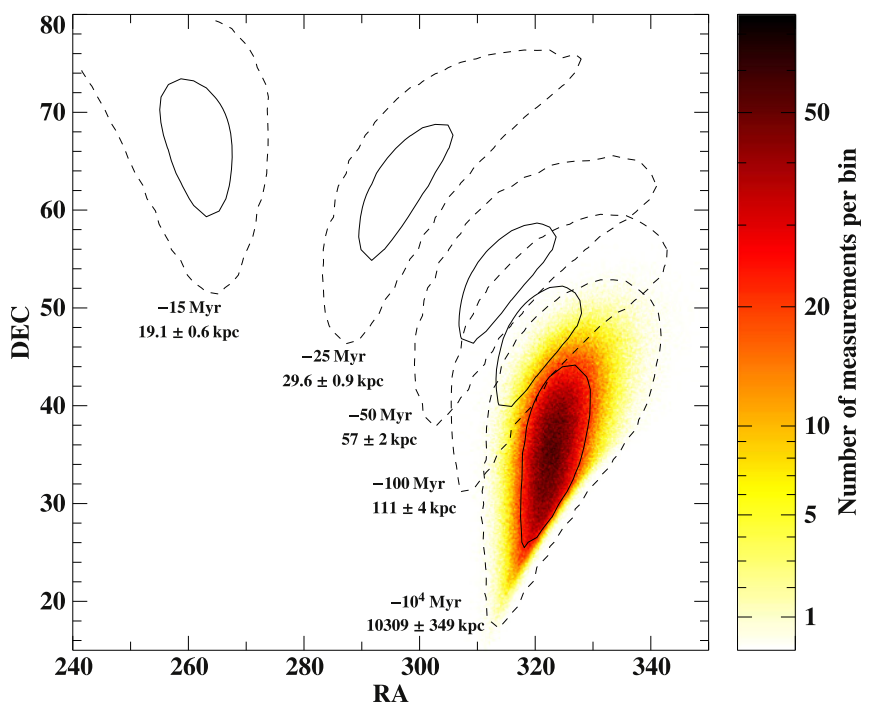

Figure 4. The sky locations and Galactic Center distances of J1211 in the past. These are calculated from the unbound orbits in the least massive Galactic mass model.

bound to the Galaxy. Model-I gave a probability of bound trajectories of $62.4 \%$. In Figure 3 we show that an origin of the system from the Galactic Center can be excluded consistently for all three models. The current location of J1211 is very close to the perigalacticon point of its orbit in the most massive model. We list the parameters of this orbit in Table 1. Figure 4 shows the past locations of J1211 in the equatorial coordinate system assuming an unbound orbit.

Gaia (Perryman et al. 2001) will provide parallax measurements for J1211 $(G=17.96, V-I=-0.013 \mathrm{mag})$, with a standard error of $\sigma_{\pi}=132 \mu \mathrm{as}$. With its $\pi=182_{-15}^{+18} \mu \mathrm{as}$ parallax (from $d=5.5 \pm 0.5 \mathrm{kpc}$ ) $\mathrm{J} 1211$ is too faint and too far for Gaia to improve on our distance measurement. However, with the anticipated $80 \mu$ as end-of-mission proper motion standard error Gaia will provide about 20 times lower error for the proper motion and improve our kinematic parameters and bound probabilities.

\section{CONCLUSIONS}

Our analysis has lowered the distance and tangential velocity of J1211 by $2 \sigma$ compared to Tillich et al. (2011). Thanks to the new radial velocity measurements and improved distance we also achieved a lower uncertainty on the Galactic rest-frame velocity. The unexpected discovery of a cool companion in a wide orbit around J1211 has severe consequences when trying to explain the extreme kinematics of this object. Along with the $\mathrm{SN}$ ejection scenario, which predicts the ejection of a single hot subdwarf star, essentially all other acceleration mechanisms discussed for HVS and runaway stars can be immediately excluded. A wide binary system like J1211 is too fragile to survive any kind of close encounter with another object that might lead to such an acceleration. A close encounter with a supermassive or intermediate-mass black hole would rip the binary apart. The same would happen during a dynamical interaction in a dense stellar population or could be caused by the kick of a core-collapse SN. Only very close binaries might survive such interactions. A globular cluster tidal tail origin is not likely either, as it would require a high-velocity cluster, and due to the low binary fraction in globular clusters we would expect more single HVS stars to be on similar orbits than binaries.

Should J1211 be unbound to the Galaxy, the origin of the system is an interesting question. Half of the known HVS stars concentrate in the direction of the constellation Leo (Brown et al. 2014). J1211 is in this direction, but its velocity vector is perpendicular to those of the known early-type HVS stars, suggesting a different origin. Although we could not associate J1211 with any of the known stellar streams, it may be the first evolved HVS system discovered from the tidal debris of a disrupted dwarf satellite galaxy.

If J1211 has a bound orbit, it must have formed with the Galaxy or accreted later. Due to the extreme kinematics of the system, a bound orbit requires a massive dark matter halo, therefore J1211 is particularly well suited to probe the Galactic potential and constrain the mass of the dark matter halo as described in Przybilla et al. (2010).

We conclude that the kinematic properties of J1211 are most likely primordial and that the binary must be an extreme halo object, which was either born in the halo population or accreted later from the debris of a destroyed satellite galaxy. The low metallicity of the K-star companion is consistent with both scenarios. The progenitor of the sdB was most likely a mainsequence star more massive than the halo turnoff mass of about $0.8 M_{\odot}$. Such old population II stars orbiting through the outermost parts of our Galaxy with extreme kinematics are very rare (Tillich et al. 2010; Scholz et al. 2015).

P.N. and E.Z. were supported by the Deutsche Forschungsgemeinschaft (DFG) through grants HE1356/49-2 and HE1356/45-2, respectively. T.K. acknowledges support by the Netherlands Research School for Astronomy (NOVA). This work is based on observations at the La Silla-Paranal Observatory of the European Southern Observatory for program number 093.D-0127(A). This work is based on observations obtained at the W.M. Keck Observatory, which is operated as a scientific partnership among the California Institute of Technology, the University of California, and the National Aeronautics and Space Administration. The Observatory was made possible by the generous financial support of the W.M. Keck Foundation. This research has used the services of ASTROSERVER.ORG.

\section{REFERENCES}

Allen, C., \& Santillan, A. 1991, RMxAA, 22, 255

Asplund, M., Grevesse, N., Sauval, A. J., \& Scott, P. 2009, ARA\&A, 47, 481

Bertone, E., Buzzoni, A., Chávez, M., \& Rodríguez-Merino, L. H. 2008, A\&A, 485,823

Bianchi, L., Herald, J., Efremova, B., et al. 2011, Ap\&SS, 335, 161

Blaauw, A. 1961, BAN, 15, 265

Brown, W. R. 2015, ARA\&A, 53, 15

Brown, W. R., Geller, M. J., \& Kenyon, S. J. 2014, ApJ, 787, 89

Brown, W. R., Geller, M. J., Kenyon, S. J., \& Kurtz, M. J. 2005, ApJL, 622, L33

Brown, W. R., Geller, M. J., Kenyon, S. J., Kurtz, M. J., \& Bromley, B. C. 2007, ApJ, 660, 311

Chen, X., Han, Z., Deca, J., \& Podsiadlowski, P. 2013, MNRAS, 434, 186

Edelmann, H., Napiwotzki, R., Heber, U., Christlieb, N., \& Reimers, D. 2005, ApJL, 634, L181

Fitzpatrick, E. L. 1999, PASP, 111, 63

Geier, S., Fürst, F., Ziegerer, E., et al. 2015, Sci, 347, 1126

Han, Z., Podsiadlowski, P., Maxted, P. F. L., \& Marsh, T. R. 2003, MNRAS, 341, 669

Han, Z., Podsiadlowski, P., Maxted, P. F. L., Marsh, T. R., \& Ivanova, N. 2002, MNRAS, 336, 449

Heber, U. 2009, ARA\&A, 47, 211 
Hills, J. G. 1988, Natur, 331, 687

Hirsch, H. A., Heber, U., O’Toole, S. J., \& Bresolin, F. 2005, A\&A, 444, L61 Hubeny, I., \& Lanz, T. 1995, ApJ, 439, 875

Husser, T.-O., Wende-von Berg, S., Dreizler, S., et al. 2013, A\&A, 553, A6

Irrgang, A., Wilcox, B., Tucker, E., \& Schiefelbein, L. 2013, A\&A, 549, A137 Lanz, T., \& Hubeny, I. 2007, ApJS, 169, 83

Lawrence, A., Warren, S. J., Almaini, O., et al. 2007, MNRAS, 379, 1599

Navarro, J. F., Frenk, C. S., \& White, S. D. M. 1997, ApJ, 490, 493

Németh, P., Kawka, A., \& Vennes, S. 2012, MNRAS, 427, 2180
Perryman, M. A. C., de Boer, K. S., Gilmore, G., et al. 2001, A\&A, 369, 339

Przybilla, N., Tillich, A., Heber, U., \& Scholz, R.-D. 2010, ApJ, 718, 37

Sakamoto, T., Chiba, M., \& Beers, T. C. 2003, A\&A, 397, 899

Scholz, R.-D., Heber, U., Heuser, C., et al. 2015, A\&A, 574, 96

Tillich, A., Heber, U., Geier, S., et al. 2011, A\&A, 527, 137

Tillich, A., Przybilla, N., Scholz, R.-D., \& Heber, U. 2010, A\&A, 517, 36

Vos, J., Østensen, R. H., Németh, P., et al. 2013, A\&A, 559, 54

Wilkinson, M. I., \& Evans, N. W. 1999, MNRAS, 310, 645 